\title{
MERCADO BURSÁTIL COLOMBIANO: UN ANÁLISIS PARA EL CONTEXTO DE BARRANQUILLA
}

\author{
Guillén León López ${ }^{1}$
}

\section{RESUMEN}

El propósito de esta investigación fue determinar el nivel de conocimiento y participación de los ciudadanos de Barranquilla respecto al mercado bursátil colombiano, de tal manera que se pueda caracterizar el perfil inversionista a partir de información complementaria de nivel socio económico y financiero. La literatura y trabajos que analicen en detalle el comportamiento de micro agentes del mercado bursátil es relativamente escasa, por tanto, los resultados de esta investigación suponen un avance en este campo. La indagación empírica y aplicación de un instrumento diseñado para este fin, permitió identificar los factores que influyen en el desarrollo y limitación de este mercado. Los resultados revelan una mayor apropiación del conocimiento bursátil entre ciudadanos con mejores dotaciones económicas y formativas respecto al resto de agentes que presentan rezagos significativos. También se evidencia a nivel agregado una correlación negativa entre nivel de ingresos e inversión bursátil. En este sentido, las conclusiones generales resaltan la necesidad de establecer estrategias financieras y educativas direccionadas a promover una mayor participación que incentive y genere interés sobre los temas financieros, en particular, del mercado bursátil; de ahí la importancia de gestionar la capacitación, promover la cultura financiera y un acceso a mayores y mejores canales de inversión para los ciudadanos.

\section{PALABRAS CLAVES}

Barranquilla, Mercado bursátil, Cultura financiera, Ciudadanos

\begin{abstract}
The purpose of this research was to determine the level of knowledge and citizen participation in Barranquilla with respect to the stock market so that we can characterize the investing profile based on complementary information in a social-economical level. The literature and the papers that analyze in a thorough way the behavior of micro-agents of the stock market are relatively scarce, therefore, the results of this research suppose and advancement in this field. The empiric enquiry and the application of a tool designed for this purpose, allowed us to identify the factors that contribute with the development and limitation of this market. The results reveal a bigger appropriation in stock market knowledge among citizens with a better financial and formative position with respect to the rest of the agents that represent meaningful backwardness. There is also an evident negative correlation between the level of income and stock investment. In this sense, the general conclusions underline the need to establish educational and financial strategies directed to promoting a better participation that promotes and generates interest on financial matters, particularly in the stock market; hence the importance of promoting training, and promotion of the financial culture and a better access to investment channels for the citizens.
\end{abstract}

\section{KEYWORDS}

Barranquilla, Stock Market, Financial Culture, Citizens

Depositado en febrero 12 de 2013, aprobado en mayo 09 de 2013.

* Esta investigación fue financiada en el marco de la IV convocatoria interna de proyectos de investigación de la Universidad Autonoma del Caribe, Barranquilla, Colombia.

1 Phd (c), Administrador de Empresas y Economista. Especialista en Finanzas y en Estadística, Magister en Economía. Docente investigador del Programa de Negocios y Finanzas de la Universidad Autónoma del Caribe, Barranquilla. E mail:guillenleon@gmail.com 


\section{INTRODUCCIÓN}

En el contexto financiero internacional, la interacción de distintos agentes de la economía con el mercado de capitales ha permitido a algunos países su desarrollo y maduración. Parte de este crecimiento obedece a la mayor participación e influencia que tiene en este mercado uno de sus agentes inversores; los ciudadanos, quienes desempeñan un papel importante en la asignación de recursos, la canalización de ahorros y destinación hacia la inversión productiva. Al servir a esta importante función, los sistemas financieros de diferentes países han evolucionado en sus estructuras, transitando desde los sistemas financieros tradicionales, a otros influenciados por los mercados financieros, con gran protagonismo del mercado bursátil. Para algunas economías desarrolladas, este mercado constituye un sector estratégico en la oferta de productos de inversión y financiación, que se favorece por un entorno legal e institucional apropiado. Levine (2004) resalta algunas formas por la cuales el mercado de capitales puede contribuir a potenciar el crecimiento económico de una nación, como son: la producción de información sobre las empresas y contribuyendo a una mejor asignación del capital; monitoreando las firmas, una vez éstas han llevado a cabo los procesos productivos, asegurando el mejor uso de los recursos; aminorando el riesgo transversalmente (mercados accionarios) y temporalmente (mercados bancarios); movilizando ahorros y superando así el problema de la indivisibilidad de la inversión y, por último, facilitando el intercambio de bienes al contribuir con la especialización mediante el aminoramiento de los costos de transacción.

En el caso de economías emergentes, este mercado continúa en su fase de crecimiento con un importante dinamismo, pero, al mismo tiempo, marcado por un conjunto de dificultades que tienden a obstaculizar su crecimiento. Entre esas debilidades se encuentra la escasa participación de micro agentes en el mercado bursátil. Sin embargo, esta situación no es ajena a otras economías, pues en el contexto financiero internacional se ha identificado un comportamiento generalizado respecto a la limitada participación en el mercado bursátil por parte de estos agentes. En este sentido Haliassos, Michael and Lyon (1994) evidencian para las familias de Estados Unidos una participación menor al $50 \%$ en el mercado bursátil, concentrada, en su mayoría, en algún producto financiero, acciones (Survey of Consumer Finances). De igual forma, para el caso de los hogares del Reino Unido, Attanasio, Banks y Tanner (2002) encuentran que más del $75 \%$ de estos agentes no tienen inversiones directas en este mercado.

Para el caso colombiano, estudios como el documento de la "Estrategia Nacional de Educación Económica y Financiera" (2010), elaborado por FOGAFIN, Banco de la República, FOGACOOP, Ministerio de Hacienda, Ministerio de Educación y Superintendencia Financiera, revelan, entre otras cosas, la necesidad por combatir el analfabetismo financiero entre la población colombiana a partir de la promoción de programas de educación económica y financiera. En relación con este tema, se aborda la cuestión del conocimiento bursátil, como uno de los temas diagnosticado como deficiente dentro de la educación financiera.

En particular, este trabajo realiza un estudio del mercado bursátil, con el objetivo de identificar el nivel de conocimiento y participación de los ciudadanos de Barranquilla en el mercado bursátil colombiano, así como las causas subyacentes a su comportamiento, de tal manera que se pueda caracterizar el perfil inversionista a partir de información complementaria de nivel socio económico y financiero. Para ello se diseñó un instrumento que recoge información y datos que evidencia el estado de conocimiento $\mathrm{y}$ apropiación en temas asociados al mercado financiero, con incidencia en el área bursátil. Los resultados del estudio revelan la limitada participación de micro agentes en el mercado de valores colombiano; lo que supone, para el gobierno y demás instituciones conexas, seguir avanzando en propuestas y nuevas estrategias que permitan una inclusión real de todos los agentes en dicho mercado. En este sentido, la investigación desarrollada ofrece información desagregada y específica que puede servir de orientación e input a los trabajos que sobre el tema se pueden abordar. 
El trabajo está organizado de la siguiente forma: La Sección 2 presenta una revisión de la literatura sobre el mercado bursátil. La Sección 3 describe la metodología utilizada. La Sección 4 aborda el análisis y tratamiento estadístico de los datos. Por último, los principales resultados y conclusiones del trabajo se recogen en la Sección 5.

\section{REVISIÓN DE LITERATURA}

Los nuevos escenarios del mercado global financiero, caracterizados por una mayor afluencia de capitales y riesgos asociados, develan la necesidad de contar con mercados internos más organizados, más profundos, más estables $\mathrm{y}$, en general, más participativos, donde los diferentes agentes de la economía puedan interactuar. En este sentido, el mercado de capitales constituye un centro de inversión de gran auge en la economía mundial, estimado en unos 36,6 trillones de dólares en la actualidad (Bespoke Investiment Group), y el cual presenta desempeños notables en las economías emergentes ${ }^{2}$. Para el caso de los mercados financieros modernos, los cambios en los roles de inversión se caracterizan por un gran protagonismo del público y otros agentes complementarios al sistema tradicional bancario. Análogamente sucede con los emisores, pues cualquier entidad con participación en el mercado puede desempeñar el rol de emisor, ya sea como entidad comercial, institución gubernamental del orden nacional e incluso internacional, como en el caso del Banco Mundial. Como consecuencia de esta gran diversidad de agentes emisores ${ }^{3}$ el mercado ha incrementado sus volúmenes de negociación, ${ }^{4}$ donde es muy importante el papel de Estados Unidos en el desarrollo de este mercado con sus bolsas, NYSE y NASDAQ, las cuales mueven entre US $\$ 300$ y US $\$ 500$ millones. Otras cotizaciones como el Eurostox 50 suman cerca de US $\$ 1,17$ billones y el Nikkei225 otros US\$1,71 billones. Nada más con estos cuatro índices y sólo una parte de las operaciones y empresas cotizantes, se obtiene un volumen corriente cercano a los US\$5.05 billones. Esta profundidad en los mercados permite avanzar hacia contextos más incluyentes y participativos, además de constituir una fuente importante para obtener recursos, como sucedió con NASDAQ, entre 1997 y 2000, con la participación de 1.649 empresas públicas que en conjunto generaron $\$ 316.5$ billones de dólares.

En este sentido, en la mayoría de países desarrollados el proceso de democratización del mercado de capitales ha sido muy activo, evidenciado en la mayor participación que el público destina en su ratio de ingreso/ahorro a inversión bursátil. En el 2008, en estas economías, la gente ya destinaba una mayor proporción de sus activos financieros al mercado de capitales, 7.6 puntos en promedio, respecto a la participación de activos financieros en la banca tradicional. De esta forma, las familias obtenían rentabilidades superiores (entre un 50\% y $60 \%$ ) en relación a las reportadas por el sistema bancario (5\%); y que varía en función del país, tipo de inversión y plazos, además de otras variables.

Esta evolución se registra para países como Estados Unidos, Francia, Italia, Suecia, Inglaterra o Alemania quienes en la década de los ochenta destinaban una proporción muy pequeña de su ahorro al mercado de capitales (5\% - 13\%); y que para el 2010 se incrementaron significativamente $(18 \%$ - 36\%). De tal forma, el crecimiento económico experimentado por estos países en ese periodo, se ha visto poderosamente influenciado por el perfeccionamiento de sus respectivos mercados de capitales y la apertura a los mismos. Luengnaruemitchai y Ong (2005) señalan que la apertura del mercado de capitales a inversionistas extranjeros constituye un factor importante para ampliar la demanda por deuda privada local; aunque reconocen que la

\footnotetext{
2 La bolsa de Brasil, en el 2013, registró un nivel de capitalización bursátil de 1020.455,3 millones de dólares, respecto a Colombia que fue de 202.693,2 de dólares

3 En 2007 en la bolsa de valores de Toronto "TSX" existían 1.613 empresas, sin ser una de la más representativa del mercado de capitales internacional.

4 En 2004, según el índice Dow Jones, el promedio diario de negociaciones tránsito de doscientos veinte mil millones de dólares a trescientos veinte mil millones de dólares en el primer bimestre de 2008. Además, ha sido imparable el crecimiento de este índice, que se multiplicó por 299 veces en sus 112 años de historia con un rendimiento anual promedio del 5\%, a pesar de la crisis del 29. Para el caso de la bolsa de Tokio, en marzo de 2010, se negociaron cerca de 2000 millones de acciones.
} 
liberalización de los mercados de capitales por sí misma es una condición insuficiente para su desarrollo; como sucede en el caso de Corea, donde a pesar de la apertura a inversionistas extranjeros, su participación en el mercado no resulta significativa.

También es cierto que estos logros responden a un proceso evolutivo del mercado, y que en todo caso han sido acompañados de políticas públicas e instituciones diseñadas para su desarrollo. Para Sungur, existen una serie de factores claves que deben coexistir para que el mercado de capitales se desarrolle y evolucione al nivel de países desarrollados; mercados de deuda pública y corporativa contribuyen en su desarrollo ${ }^{5}$ sobre la base de instituciones solidas. En países emergentes el surgimiento de la deuda corporativa resultó de la necesidad de encontrar fuentes alternas de financiamiento ante la crisis financiera internacional.

Para el caso de Colombia, el interés por desarrollar el mercado de capitales se ha mantenido, a pesar de ser un proceso lento, debido a su larga transición de país pobre $(1950$ - 1970) a país de renta media (1980 - 2000), confluyendo en su momento con una débil legislación regulatoria en el ámbito financiero y de valores (González, A. Rojas , C. 2008). Sin embargo, y pese a un crecimiento económico notable en la última década (3.5\% promedio), persisten los obstáculos para su progreso, y la discusión acerca de por qué no se ha desarrollado el mercado de capitales en Colombia permanece tan vigente como hace unas décadas, como lo evidenciaron en la década de los noventa los estudios elaborados por la Misión de Estudios del Mercado de Capitales (Ministerio de Hacienda et al. 1996). Factores como su baja liquidez y participación limitada (pocos emisores y bajos volúmenes transados), escasa profundidad (pocos productos financieros), costos de emisión (altos costos para emitir acciones), regulación tributaria (falta de estímulos fiscales para invertir), y, en general, una normativa que limita la expansión/ excluye del mercado a los pequeños agentes, se consideran como sus principales barreras. Dicho esto, si comparamos la capitalización bursátil de Colombia (19\%) con algunos mercados de la región, Chile (130\%), Brasil (104\%) y México $(45 \%)^{6}$, encontramos que los desafíos aún son muy grandes en materia del desarrollo de un mercado de capitales, en contraste con el crecimiento económico experimentado.

A pesar de ello, desde la década de los setenta, se vienen realizando foros de discusión académica, con participación del gobierno y el sector financiero, y uno de los más destacados es el conocido "Simposio del Mercado de Capitales" Los simposios de los años ochenta sugerían que, a pesar de los avances académicos, las emisiones primarias y la bursatilidad de papeles del sector privado continuaban siendo bajos. Según la Asociación nacional de instituciones financieras los factores que estructuralmente han impedido un desarrollo profundo del mercado de capitales en Colombia son: 1) el deseo de mantener un control "cerrado" de las firmas, por temor a nuevos socios; 2) el manejo discrecional contable y tributario de muchas firmas con gran tradición familiar; y 3) la falta de transparencia y de buen Gobierno Corporativo.” (Anif, 2007)

También se destacan en el país la adopción de importantes políticas con el fin de promover el desarrollo del mercado de capitales: la ley 75 de 1986, primera ley en propiciar la eliminación de sesgos tributarios contra la emisión de acciones. De la misma forma, en la Constitución Política de 1991, la ley 35 de 1993 y la ley 964 de 2005 constituyeron marcos regulatorios importantes para acelerar el desarrollo del mercado de capitales en Colombia. En esta vía, la más reciente expedición de la Ley 1242 y la creación de la figura independiente del custodio, han contribuido al desarrollo y crecimiento de los Fondos de Inversión Colectiva (FIC) en Colombia; los cuales en el mercado mundial movilizan US $\$ 25,59$ trillones, representados en un total de 73.343 fondos, de los cuales Estados Uni-

\footnotetext{
5 En el 2006, el tamaño del bond market a nivel internacional se estimaba en 44,9 trillones de dólares, de los cuales 25,2 trillones hacían parte del bond market estadounidense.

6 Datos obtenidos de World Economic Outlook Database y el World Federation of Exchanges.

7 Evento organizado por Asobancaria, desde hace 26 años.
} 
dos congrega más de la mitad. En este país, el $46.3 \%$ de los hogares invierte en fondos de inversión (En Colombia es del 2\%) que representa cerca del $86 \%$ del PIB americano. En Brasil representan el 51,5\% (13935 fondos), México, $10,1 \%$ (565 fondos) y Colombia solo un 6,8\% (238 fondos) del PIB. (Dinero, julio de 2014).

No obstante, estudios recientes revelan para Colombia unos altos costos de capital accionario y unos grados de competitividad bajos frente a otras fuentes de financiamiento. En este sentido, la realidad del mercado nos sugiere que la pobre inscripción de emisores de acciones en el mercado y la alta concentración de las negociaciones en pocas acciones, no ha cambiado en casi 20 años. Adicionalmente, otros estudios señalan que el tamaño promedio de firmas cotizantes frente al resto de Latinoamérica continúa siendo reducido, sumado a la escasa diversificación de los sectores que participan en dicho mercado accionario, lo cual evidencia no solo la concentración, sino la exclusión del mismo mercado, pues el número de empresas que tienen acceso es muy bajo, teniendo en la actualidad hay un listado de 140 emisores en la Bolsa de Valores de Colombia, de 27.541 empresas que registran sus estados financieros ante las entidades de vigilancia y control (Superintendencias). Estas cifras indican que sólo el $0,3 \%$ de las empresas realizan algún tipo de actividad en el mercado de capitales colombiano.

Este panorama desalentador en el sector empresarial, se agudiza mucho más en el ámbito ciudadano, donde la participación resulta más rezagada, - pese a los esfuerzos y avances por democratizar el mercado bursátil - en comparación con otros contextos donde los niveles de participación de los ciudadanos exhiben mejores desempeños ${ }^{8}$. Factores asociados al desco- nocimiento y desinformación generalizada en temas financieros, restricciones de ingresos y ahorros ${ }^{9}$, estímulos tributarios, escasa cultura bursátil, aversión al riesgo, asimetría de información, entro otros, constituyen obstáculos que limitan la participación activa y responsable de ciudadanos en este segmento de la economía. Es por ello, que el gobierno en alianza con la bolsa de valores de Colombia, universidades, firmas comisionistas de bolsa, fiduciarias ${ }^{10}$, bancos y empresas, realizan programas, capacitaciones y diferentes estrategias de promoción e inversión para que la población interactúe y se involucre más con los mercados financieros, en particular el mercado bursátil colombiano ${ }^{11}$. En ese sentido, la ley del mercado de valores (964 de 2005) provee una estructura única de regulación que ofrece estabilidad jurídica y reglamenta las actuaciones del gobierno, además de establecer un mecanismo legal para promover los derechos de los accionistas minoritarios, la competencia y transparencia de este mercado. En este aspecto, la configuración de una cultura bursátil y un sistema financiero que logre permear estos comportamientos resultan fundamentales para el desarrollo del mercado de capitales, pues a junio del 2014, el saldo en CDT acumulaba $\$ 121$ billones, $15 \%$ más que el 2013, y las cuentas de ahorro en bancos comerciales ascendieron a $\$ 136$ billones, con un crecimiento anual del $17 \%$, en contraste con la disminución de inversionistas en bolsa, pues en promedio, 1 millón de ciudadanos le apuestan a este mercado respecto a los 20 millones que ostentan al menos una cuenta de ahorros.

\section{DATOS Y METODOLOGÍA}

Los datos utilizados se obtuvieron de forma primaria, a partir del trabajo de campo realizado en distintas localidades de la ciudad de Barranquilla. La metodología para este estudio es de

\footnotetext{
8 En España una cuarta parte de la propiedad de las acciones de empresas españolas cotizadas estaba en manos de las familias españolas al cierre del ejercicio 2012. En particular, el 25,1\% de los títulos era de su propiedad, prácticamente cuatro puntos porcentuales más que el 2011, según el informe sobre la Distribución de la Propiedad de las Acciones Españolas Cotizadas del Servicio de Estudios de Bolsas y Mercados Españoles (BME)

9 Un $60 \%$ de la población de Colombia tiene ingresos entre 2 y 3 SMMLV con un nivel de ahorro y deuda del $20 \%$ 10 En este año Asofiduciarias, lanzó la campaña denominada: Plata Trabajando, como contribución a la educación financiera del país, con el fin de canalizar recursos de ahorradores hacia los fondos de inversión colectiva.

11 La ley 1328 de 2009 (Reforma Financiera) estableció que las entidades del sector financiero deben desarrollar programas de educación económica y financiera; dicha consagración se hizo como un Principio General y como una obligación especial a cargo de las entidades vigiladas por la Superintendencia Financiera de Colombia y hace relación al desarrollo de programas educativos.
} 
carácter descriptivo explicativo y fue planteado diferencialmente, de acuerdo con el propósito a abordar, de la siguiente manera: La investigación se llevó a cabo en tres fases, en la primera se validaron y ajustaron los interrogantes del instrumento de investigación (prueba piloto); posteriormente se recolectaron y sistematizaron los datos en base a toda la muestra (trabajo de campo) y, en la tercera fase (análisis), se revisaron, estudiaron y prepararon los resultados que se consolidan en este documento.

Para lograr la caracterización del perfil inversionista de los ciudadanos de Barranquilla se diseñó un instrumento en forma de encuesta con preguntas cerradas de selección múltiple, de forma dicotómica, escalar y de valoración, aplicadas de manera presencial y aleatoriamente a personas mayores de 18 años, distribuidas en las cinco localidades de la ciudad ${ }^{12}$. En total se realizaron 650 encuestas, de las cuales fueron descartadas 125 por presentar sesgos estadísticos. Las 525 encuestas finales (105 por localidad) con una tasa de respuesta del 100 $\%$, intentan capturar información relevante de distintas características del ciudadano y su situación financiera; para ello la encuesta se divide en tres secciones. En la primera sección se explora sobre datos demográficos y sociales. La segunda aborda información económica y costumbres financieras. En la tercera sección, para quienes aplican en la pregunta - filtro sobre conocimiento bursátil; se continúa con el estudio que examina el nivel de apropiación y conocimiento sobre el mercado bursátil colombiano. El siguiente cuadro resume el tratamiento metodológico abordado en esta investigación.

\section{RESULTADOS}

En la línea de los objetivos trazados en la investigación se organizan los resultados, de tal forma, que en la primera parte (preguntas 1 - 9), se establece el perfil demográfico y socio económico de los ciudadanos. La segunda parte, preguntas $(10-23)$, está direccionada a obtener información económica y costumbres
Cuadro 1. Marco metodológico

\begin{tabular}{|c|c|}
\hline $\begin{array}{l}\text { Metodología de } \\
\text { selección de la } \\
\text { muestra }\end{array}$ & $\begin{array}{l}\text { Probabilística estratificada, } \\
\text { con selección aleatoria, } \\
\text { garantizando, representación } \\
\text { balanceada de todas las } \\
\text { características del universo de } \\
\text { estudio, fijación anticipada de } \\
\text { márgenes de precisión (error), } \\
\text { y la posibilidad de calcular } \\
\text { a posteriori la precisión } \\
\text { efectivamente observada en } \\
\text { los resultados. }\end{array}$ \\
\hline Población objetivo & $\begin{array}{l}\text { Hombres y mujeres mayores } \\
\text { de } 18\end{array}$ \\
\hline $\begin{array}{l}\text { Universo } \\
\text { representado }\end{array}$ & $\begin{array}{l}\text { Ciudadanos de la ciudad de } \\
\text { Barranquilla }\end{array}$ \\
\hline Técnica & $\begin{array}{l}\text { Entrevista personalizada con } \\
\text { aplicación de un cuestionario } \\
\text { estructurado. }\end{array}$ \\
\hline $\begin{array}{l}\text { Tamaño de } \\
\text { muestra }\end{array}$ & $\begin{array}{l}650 \text { encuestas distribuidas } \\
\text { uniformemente en las } 5 \\
\text { localidades de la ciudad ( } 130 \\
\text { p/u) }\end{array}$ \\
\hline $\begin{array}{l}\text { Momento } \\
\text { estadístico }\end{array}$ & $\begin{array}{l}\text { Septiembre a diciembre de } \\
2013\end{array}$ \\
\hline Financiación & $\begin{array}{l}\text { IV Convocatoria institucional } \\
\text { para el financiamiento de } \\
\text { proyectos de investigación de } \\
\text { la Universidad Autónoma del } \\
\text { Caribe }\end{array}$ \\
\hline $\begin{array}{l}\text { Margen de error } \\
\text { observado }\end{array}$ & $\begin{array}{l}2,9 \% \text { total (Error estándar } \\
\text { relativo máximo, para } \\
\text { fenómenos presentes en la } \\
\text { población con una frecuencia } \\
\text { de ocurrencia del } 50 \% \text { y un } \\
\text { nivel de confianza del } 95 \% \text {. }\end{array}$ \\
\hline Cubrimiento & $\begin{array}{l}\text { Cinco localidades de } \\
\text { la ciudad: Suroriente, } \\
\text { Suroccidente, Área } \\
\text { Metropolitana, } \\
\text { Norte Centro Histórico, y } \\
\text { Riomar }\end{array}$ \\
\hline
\end{tabular}

12 Según el acuerdo distrital No. 006 de 2006, la ciudad se encuentra conformada en 5 localidades: Suroriente, Suroccidente, Área Metropolitana, Norte Centro Histórico y Riomar. 
financieras. Por último, el tercer apartado hace énfasis en el propósito central de la investigación, indagando sobre el nivel de conocimiento que los ciudadanos tienen del mercado bursátil colombiano (Preguntas $24-47$ ).

La caracterización socioeconómica permitió establecer el sexo, la edad, el estado civil, el estrato socio económico, el nivel educativo, el número de miembros en el hogar, entre otras variables. El 80\% de los encuestados se encuentran entre los 18 y 50 años de edad. La situación laboral entre los hombres y mujeres encuestados es muy similar, lo cual no sucede a nivel de estratos, evidenciándose diferencias significativas en los niveles y las formas de empleo.

Tabla No 1. Distribución por actividad total, para cada estrato y género.

\begin{tabular}{|c|c|c|c|c|c|c|c|c|}
\hline \multirow{2}{*}{ Actividad } & \multicolumn{5}{|c|}{ Estrato Socioeconómico } & \multicolumn{2}{c|}{ Género } & \multirow{2}{*}{ Total } \\
\cline { 2 - 8 } & $\mathbf{2}$ & $\mathbf{3}$ & $\mathbf{4}$ & $\mathbf{5}$ & $\mathbf{6}$ & Hombres & Mujeres & \\
\hline Trabaja & $66 \%$ & $56 \%$ & $69 \%$ & $86 \%$ & $100 \%$ & $66 \%$ & $63 \%$ & $65 \%$ \\
\hline Dependiente & $60 \%$ & $77 \%$ & $75 \%$ & $61 \%$ & $83 \%$ & $71 \%$ & $68 \%$ & $70 \%$ \\
\hline Independiente & $40 \%$ & $23 \%$ & $25 \%$ & $39 \%$ & $17 \%$ & $29 \%$ & $32 \%$ & $30 \%$ \\
\hline No trabaja & $34 \%$ & $44 \%$ & $31 \%$ & $14 \%$ & $0 \%$ & $34 \%$ & $37 \%$ & $35 \%$ \\
\hline
\end{tabular}

Fuente: cálculo del autor

Se confirma el hecho de que Barranquilla es una ciudad próspera, pero desigual. Los salarios están distribuidos de forma inequitativa, a la vez que, un poco más del $50 \%$ se concentra en los dos primeros niveles salariales (1 y 2 SMMLV). El nivel de estudios predominante corresponde al nivel de pregrado (39\%), y solo un $(5 \%)$ ostenta formación de posgrado. El núcleo familiar está conformado en su mayoría por hogares entre 3 y 5 personas $(85 \%)$.

Respecto al perfil económico, el $(79,82 \%)$ trabaja en el sector privado y se concentra en gran parte en dos sectores, servicios $(36,50 \%)$ y comercio $(24,63 \%)$. Se destaca que más de la mitad de la población encuestada devenga entre dos salarios mínimos, y sus niveles de ahorro sean del $5-15 \%$ de su ingreso, para una gran mayoría de la población encuestada $(70,7 \%)$. Este ahorro se dirige en un $70 \%$ a depósitos bancarios y un $(20 \%)$ al pago de hipotecas. Al momento de invertir las preferencias del consumidor financiero se enfocan en activos financieros $(36 \%)$, activos inmobiliarios $(26 \%)$ y ocio $(19 \%)$.

En cuanto a los hábitos financieros, el (45\%) no registra ninguna relación de planeación finan- ciera versus un (55\%) que mantiene un registro de ahorros, ingresos, gastos y deudas.

Para el tercer componente, conocimiento bursátil, los hallazgos revelan que el (61\%) carece de conocimiento sobre este mercado, y quienes lo conocen $(39 \%)$, tiene una noción regular (44\%) y baja (205) del mismo. En cuanto a la inversión en bolsa, el (93\%) no la tienen, y del (7\%) que participa lo hacen en su mayoría en títulos de renta variable, acciones, con un (69\%) y renta fija, CDTs con el (28\%).

El limitado conocimiento que sobre el mercado bursátil se tiene en Barranquilla va en orden ascendente conforme se escala en los estratos. Aun así, sorprende el contraste encontrado entre estratos, pues las diferencias entre estos respecto a su desconocimiento no son muy significativas. $(25 \%)$.

Además de la falta de conocimiento $(48 \%)$, como tal, el desinterés $(25 \%)$ y la falta de dinero $(27 \%)$ constituyen las razones principales para no acudir al mercado bursátil.

En cuanto a las barreras del mercado bursátil, la falta de información $(56,54 \%)$, inestabilidad 
y riesgo (56\%), tamaño del mercado (29\%) y montos de inversión $(15,71 \%)$ se presentan como los principales obstáculos de acceso.

Resulta importante, entre quienes no conocen el mercado bursátil, las expectativas e interés por acercarse y descubrir las posibles ventajas que pueda ofrecer el mercado como una opción más de inversión $(47,62 \%)$. En este sentido, se debe fortalecer las estrategias y canales de promoción del sistema financiero, pues el $(85,33 \%)$ manifestó no conocer el programa nacional de educación económica y financiera promovido por el gobierno nacional y otros entes privados.

\section{CONCLUSIÓN}

En la actualidad algunas instituciones desarrollan campañas y programas educativos en temas económicos y financieros; sin embargo, todos estos esfuerzos se realizan de manera aislada y desarticulada con los otros, y, en su gran mayoría, tienen un radio de acción muy limitado en términos de cobertura, prueba de ello es que el (85\%) desconocen el programa de educación financiera, y por ello consideran muy importante incorporar contenidos financieros en los currículos de educación secundaria $(88 \%)$.

Factores asociados al desconocimiento y desinformación generalizada en temas financieros, restricciones de ingresos y ahorros, estímulos tributarios, escasa cultura bursátil, aversión al riesgo, asimetría de información, entro otros, constituyen obstáculos que limitan la participación activa y responsable de ciudadanos en este segmento de la economía.

\section{REFERENCIAS}

Arbeláez, M., Zuluaga, S., Guerra, M.. (2002) “El Mercado de Capitales Colombiano en los Noventa y las Firmas Comisionistas de Bolsa",Alfaomega Fedesarrollo,

Bogotá, D.C.

Clavijo, S. y A. Vera (2007) "El desarrollo del mercado de capitales en Colombia” En Carta Financiera, No. 136, noviembre-febrero, pp. 7-13.

Córdoba, J. P., R. Junguito, S. Montenegro y F. Renjifo (2009) "Perspectivas del mercado de capitales: ¿qué queda por hacer?" En Carta Financiera, No. 148, octubre-diciembre, pp. 25-28.

De la Torre, A., Gozzi, J., y Schmukler S. (2006) "Financial Development in Latin America: Big Emerging Issues, Limited Policy Answers", The World Bank Policy Research Working Paper, No. 3963.

Demirgüç-kunt, A. y Levine, R. (1995) "Stock Markets and Financial Intermediaries: Estlized Facts", The World Bank Policy Research Working Paper, No. 1462, The World Bank

Haliassos, M. and Lyon. (1994). "Progressivity of Capital Gains Taxation with Optimal Portfolio Selection” En (J.Slemrod, ed). Tax Progressivity and Income Inequality Cambridge: Cambridge University Press.

Levine, R. (2004) "Finance and Growth: Theory and Evidence”, NBER Working Papers, No. 10766.

Levine, R. (1992) "Financial Structures and Economic Development”, The World Bank Policy Research Working Paper, No. 849,

Ministerio de Hacienda, Banco Mundial y Fedesarrollo (1996) "Misión de Estudios del Mercado de Capitales: Informe Final", Mimeo, Bogotá D.C.

Sungur, O. "Limited Asset Market Participation: Evidence From Household Data”

Uribe, J. (2007). “Caracterización del mercado accionario colombiano, 2001-2006: Un análisis comparativo” Banco de la república. Borradores de economía, No. 456. 
ANEXOS

PRIMER FACTOR: INFORMACION GENERAL
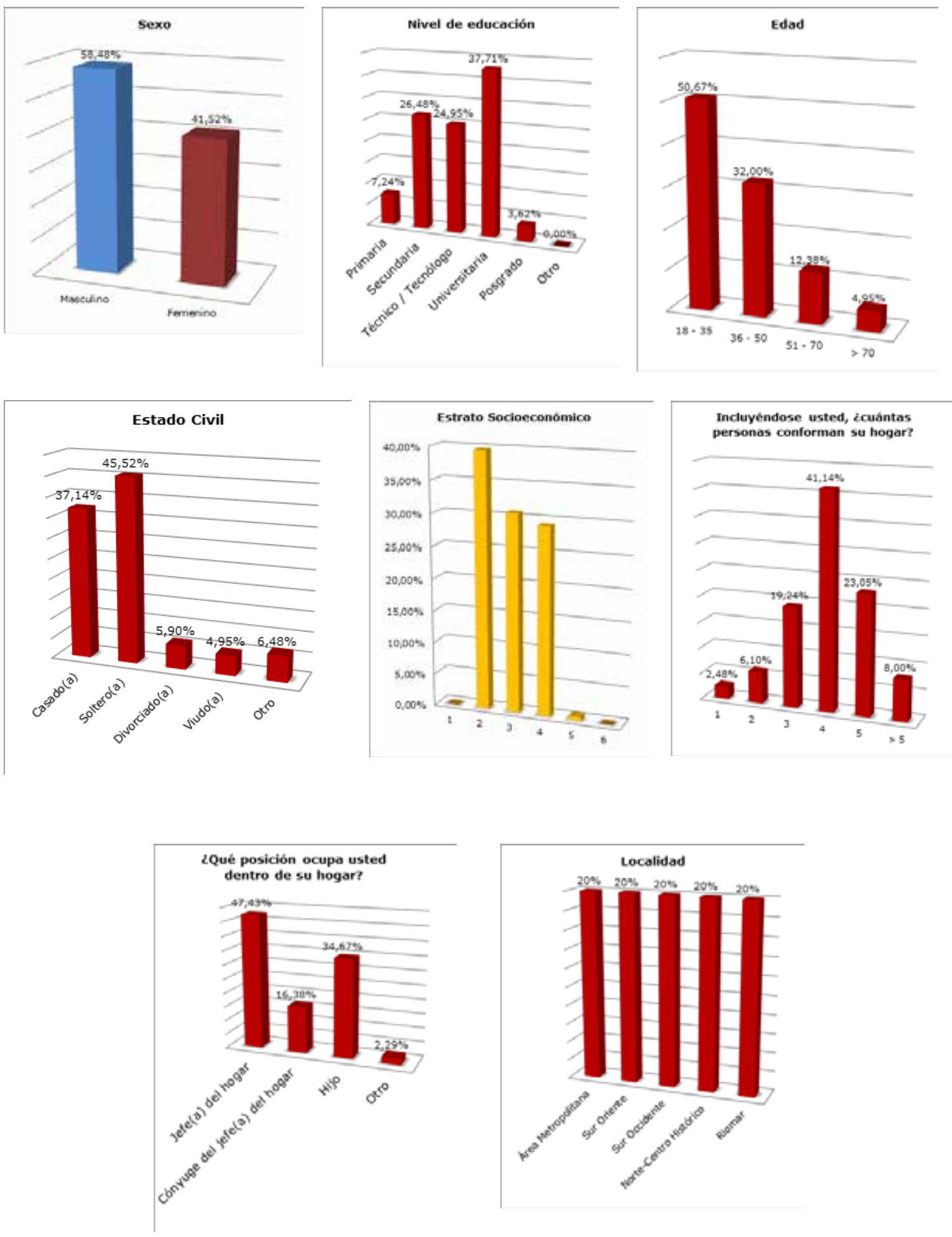


\section{SEGUNDO FACTOR: INFORMACION ECONOMICA}
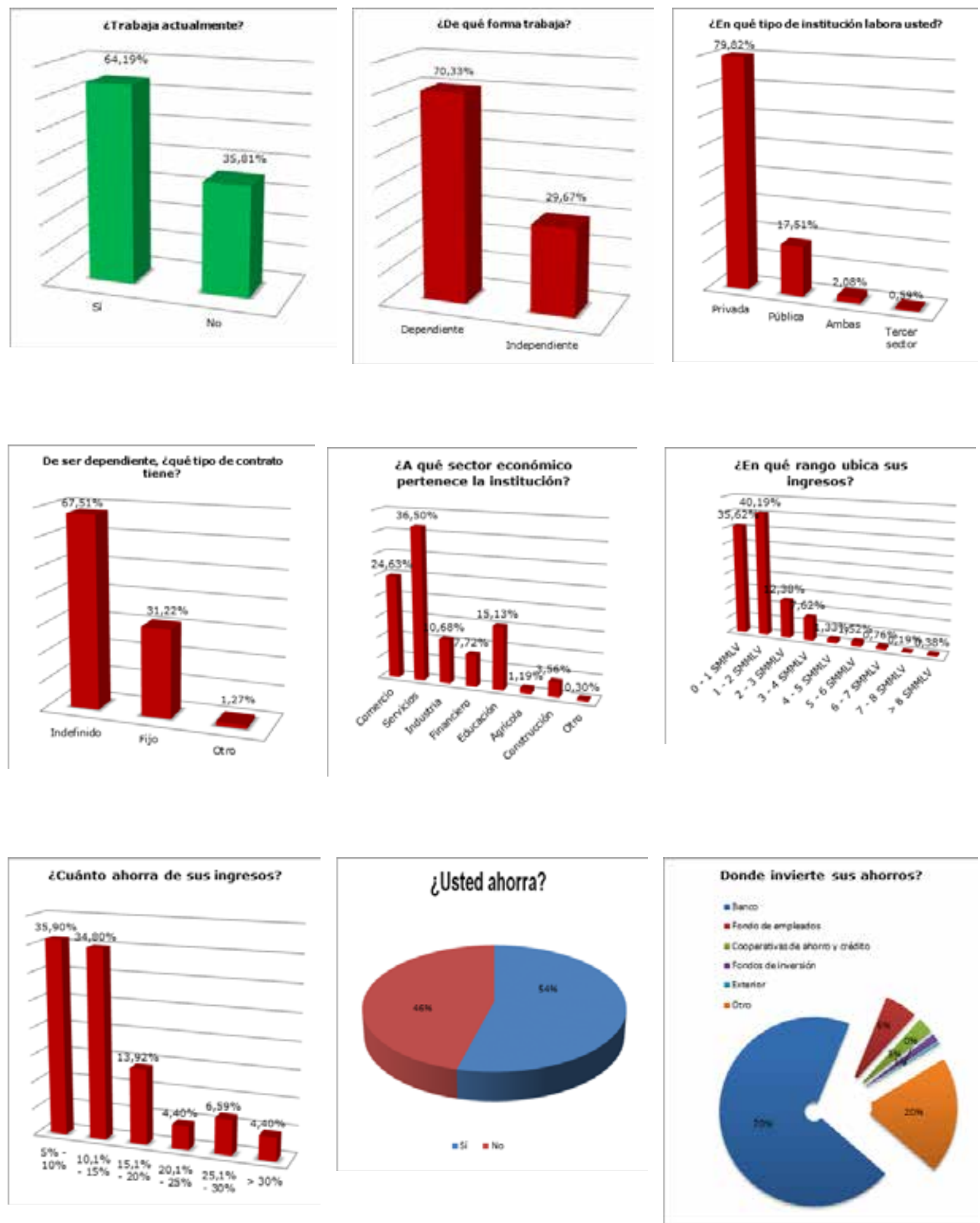

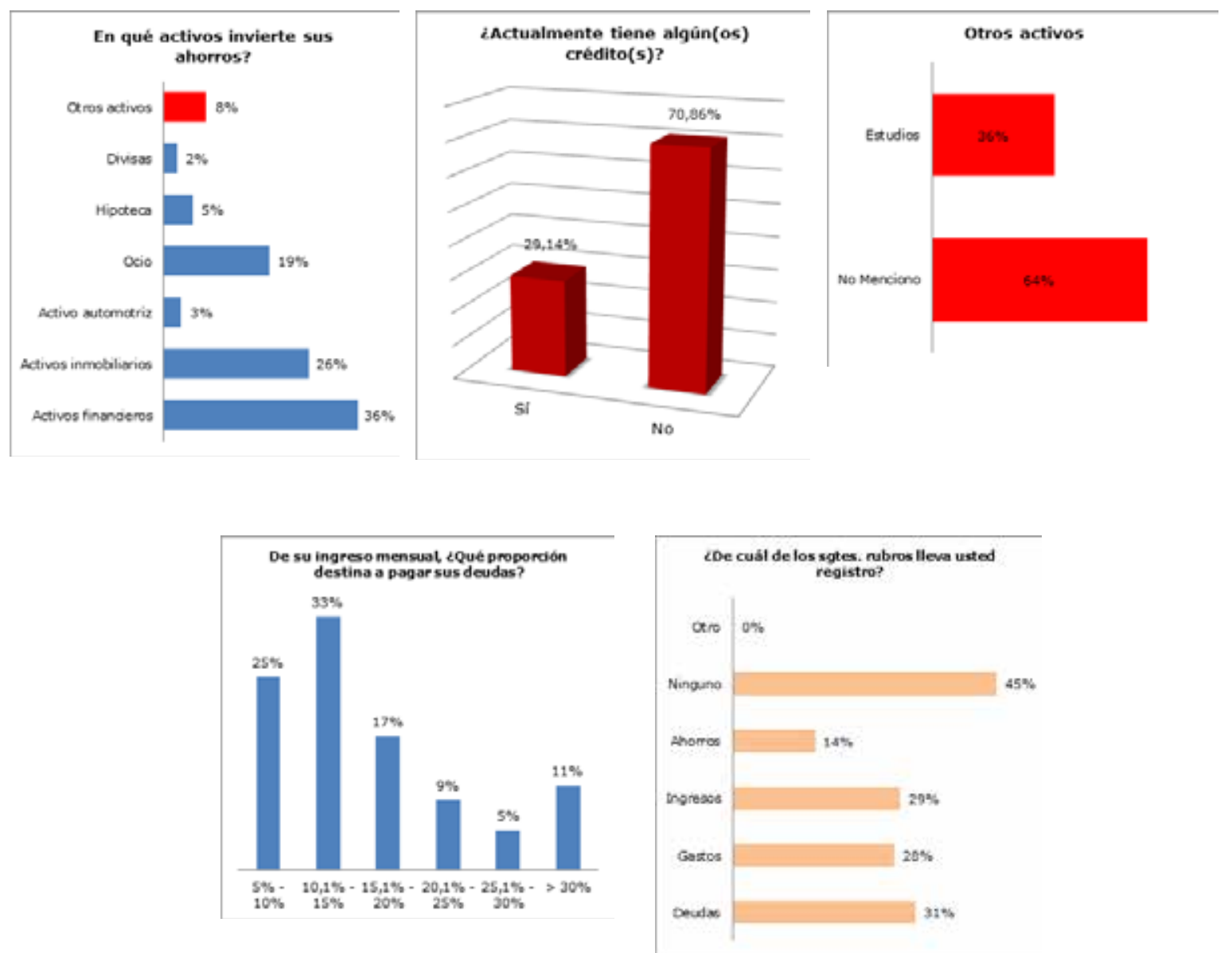

TERCER FACTOR: CONOCIMIENTO BURSATIL
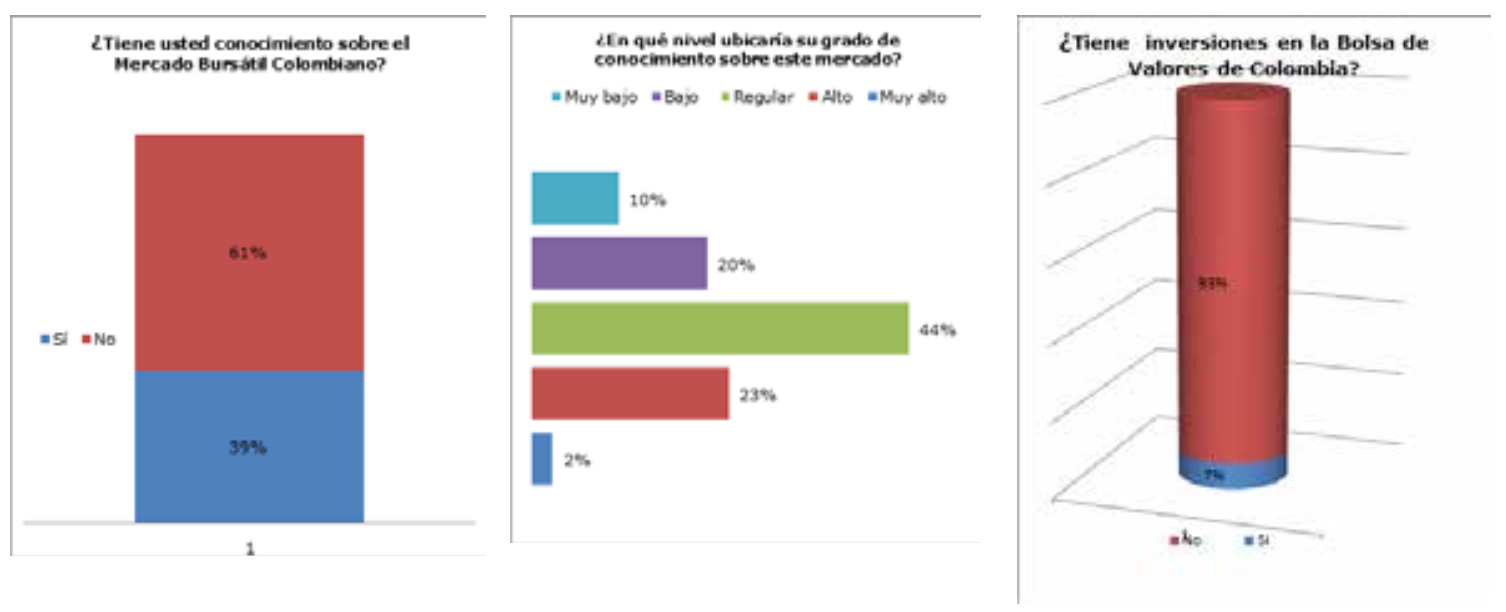
Ordene según su importancia (siendo 1 la más importante), las siguientes frases que identifican las funciones del mencado bursbtil:

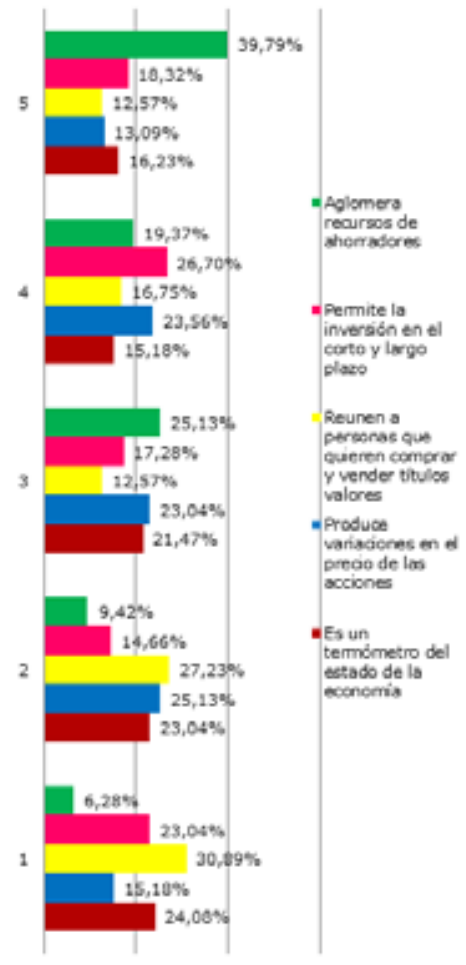

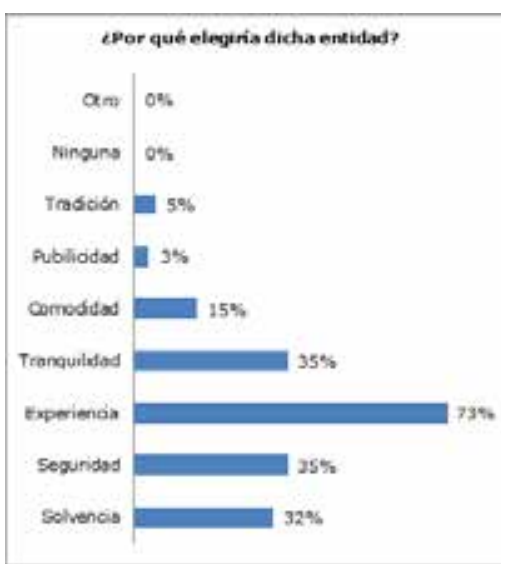

En los último seis meses. ¿Ha adquirido algún título valor emitido por el Estado alguna Empresa?
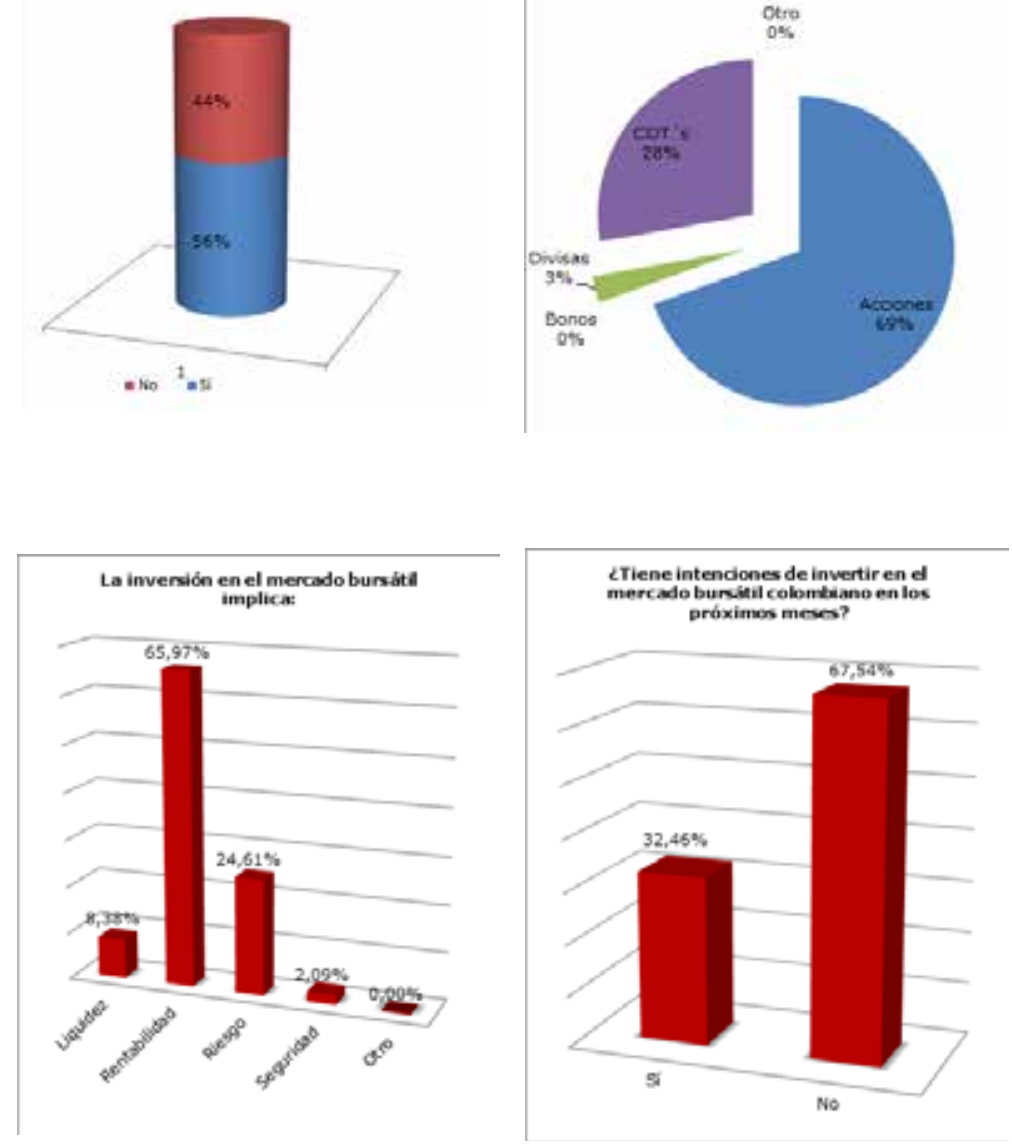
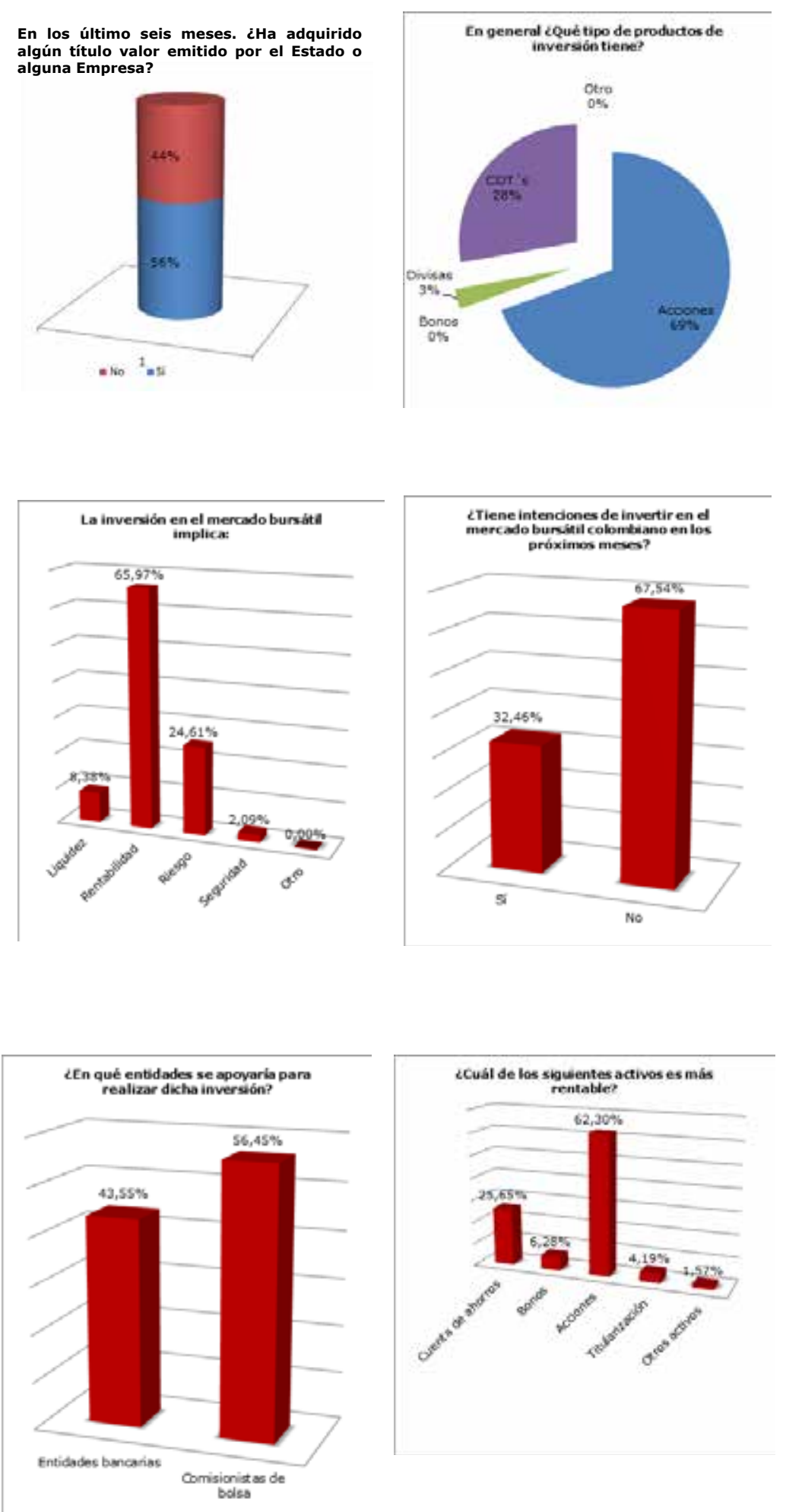

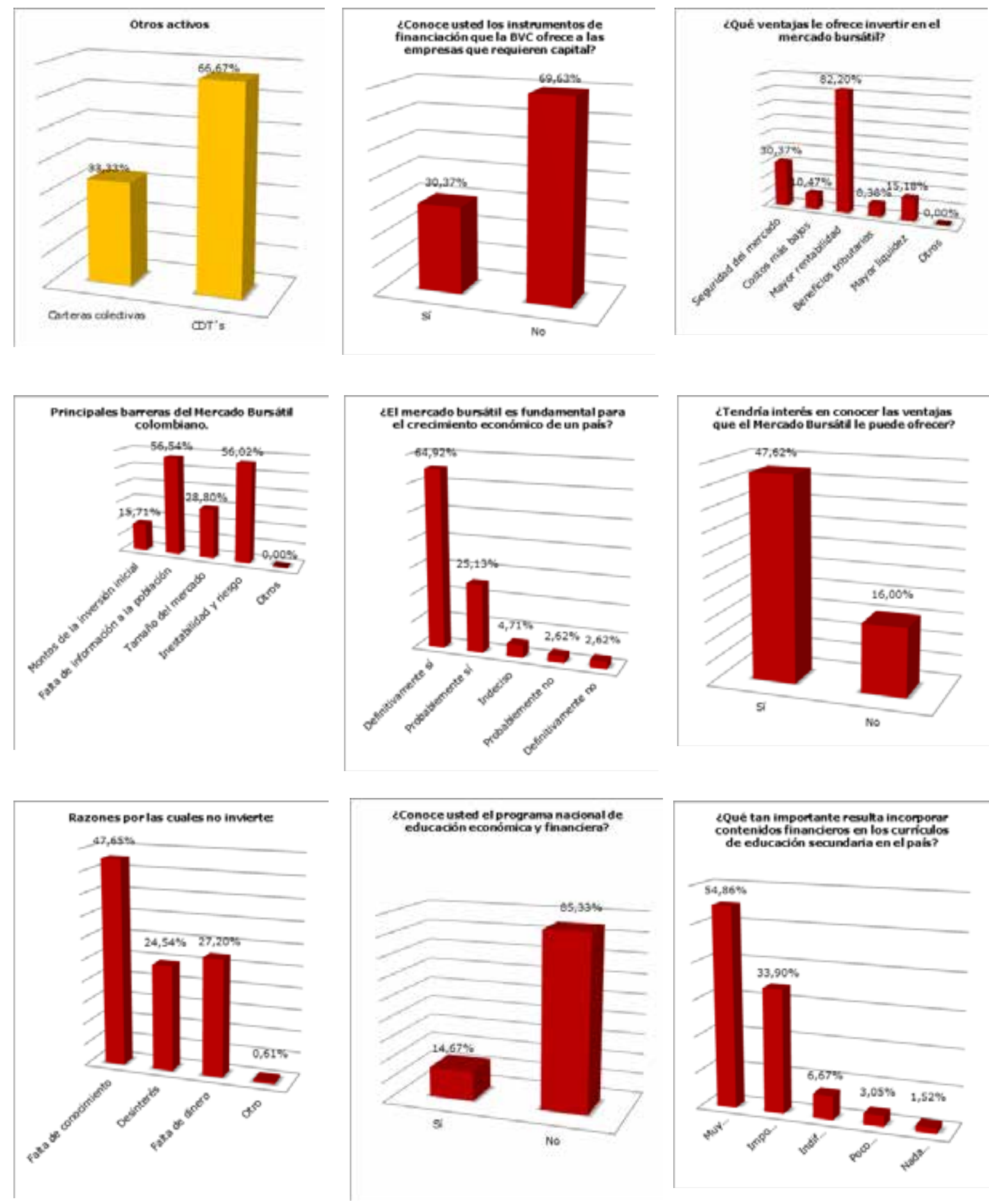

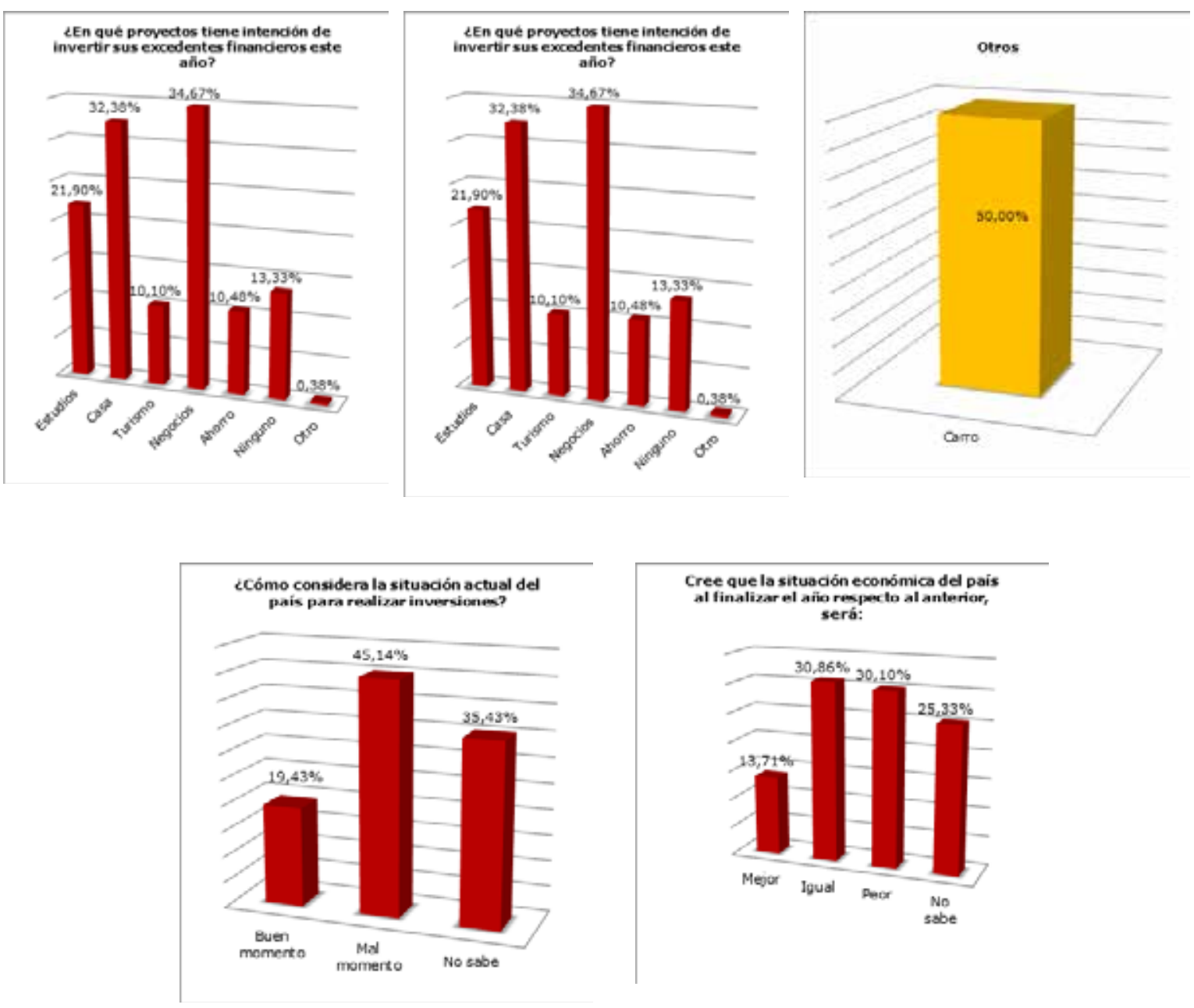